\title{
AN INEXACT PROXIMAL DECOMPOSITION METHOD FOR VARIATIONAL INEQUALITIES WITH SEPARABLE STRUCTURE
}

\author{
Erik A. Papa Quiroz ${ }^{1,2, *}$, Orlando Sarmiento ${ }^{3}$ and Paulo Roberto Oliveira ${ }^{3}$
}

\begin{abstract}
This paper presents an inexact proximal method for solving monotone variational inequality problems with a given separable structure. The proposed algorithm is a natural extension of the Proximal Multiplier Algorithm with Proximal Distances (PMAPD) proposed by Sarmiento et al. [Optimization 65 (2016) 501-537], which unified the works of Chen and Teboulle (PCPM method), and Kyono and Fukushima (NPCPMM) developed for solving convex programs with a particular separable structure. The resulting method combines the recent proximal distances theory introduced by Auslender and Teboulle [SIAM J. Optim. 16 (2006) 697-725] with a decomposition method given by Chen and Teboulle for convex problems and extends the results of the Entropic Proximal Decomposition Method proposed by Auslender and Teboulle, which used to Logarithmic Quadratic proximal distances. Under some mild assumptions on the problem we prove a global convergence of the primal-dual sequences produced by the algorithm.
\end{abstract}

Mathematics Subject Classification. 90C33.

Received August 15, 2018. Accepted February 19, 2020.

\section{INTRODUCTION}

Let $T: \mathbb{R}^{n} \times \mathbb{R}^{p} \rightrightarrows \mathbb{R}^{n} \times \mathbb{R}^{p}$ be a maximal monotone operator and let

$$
\Omega:=\{(x, z) \in \overline{\mathrm{C}} \times \overline{\mathrm{K}}: A x+B z=b\}
$$

where $\mathrm{C} \subset \mathbb{R}^{n}$ and $\mathrm{K} \subset \mathbb{R}^{p}$ are nonempty open convex sets, $\overline{\mathrm{C}}$ and $\overline{\mathrm{K}}$ denote the closure (in the Euclidean topology) of $\mathrm{C}$ and $\mathrm{K}$ respectively, $A \in \mathbb{R}^{m \times n}, B \in \mathbb{R}^{m \times p}$ and $b \in \mathbb{R}^{m}$. This paper is interested for solving the following variational inequality problem with separate structure, denoted from now on as $\operatorname{VI}(\Omega, T)$ problem: to find a pair $\left(x^{*}, z^{*}\right) \in \Omega$ and $g^{*}:=\left(g_{1}^{*}, g_{2}^{*}\right) \in T\left(x^{*}, z^{*}\right)$ such that

$$
\left\langle x-x^{*}, g_{1}^{*}\right\rangle+\left\langle z-z^{*}, g_{2}^{*}\right\rangle \geq 0, \forall(x, z) \in \Omega,
$$

where $\langle\cdot, \cdot\rangle$ denotes the inner product in the appropriate Euclidean space.

Keywords. Variational inequalities, maximal monotone operators, separable structure, proximal distances.

1 Universidad Nacional del Callao, Callao, Perú.

2 Universidad Privada del Norte, Trujillo, Perú.

3 Systems Engineering and Computer Science Program, COPPE, Federal University of Rio de Janeiro, CP 68511, Rio de Janeiro, RJ 21941-972, Brazil.

${ }^{*}$ Corresponding author: erikpapa@gmail.com 
Some papers devoted to solve the above problem and several applications in networks economics, transportation equilibrium problems, etc., can be found for example in $[1,2,6,11,13,15,17,23]$ and references therein.

Throughout, we assume that the $\operatorname{VI}(T, \Omega)$ problem has a solution and $\operatorname{dom}(T) \cap(\mathrm{C} \times \mathrm{K}) \neq \emptyset$.

Then, it can be easily verified that $\left(x^{*}, z^{*}\right)$ solves the $\operatorname{VI}(T, \Omega)$ problem, if and only if, there exists $y^{*} \in \mathbb{R}^{m}$, playing the role of a dual multiplier for the constraint (1.1), such that $\left(x^{*}, z^{*}, y^{*}\right)$ solves the following primal-dual formulation: find $\left(x^{*}, z^{*}, y^{*}\right) \in \overline{\mathrm{C}} \times \overline{\mathrm{K}} \times \mathbb{R}^{m}$ and $g^{*}:=\left(g_{1}^{*}, g_{2}^{*}\right) \in T\left(x^{*}, z^{*}\right)$ such that

$$
\begin{aligned}
\left\langle x-x^{*}, g_{1}^{*}+A^{T} y^{*}\right\rangle+\left\langle z-z^{*}, g_{2}^{*}+B^{T} y^{*}\right\rangle & \geq 0, \forall(x, z) \in \overline{\mathrm{C}} \times \overline{\mathrm{K}}, \\
A x^{*}+B z^{*} & =b .
\end{aligned}
$$

For solving the $\operatorname{VI}(T, \Omega)$ problem, we propose an inexact proximal decomposition algorithm using proximal distances, which combine the recent proximal distances theory introduced by Auslender and Teboulle [3] with the Entropic Proximal Decomposition Method proposed in [1]. This scheme is in fact an extension of Chen and Teboulle's method [9] (which was developed for solving convex programs with a particular separable structure) and the Entropic Proximal Decomposition Method.

The extension is in two directions. Firstly we consider the more general framework of variational inequalities with convex constraints and secondly we use here the recent proximal distance theory introduced by Auslender and Teboulle [3] in place of the usual quadratic proximal theory (for the case of the Chen and Teboulle method) and Logarithmic Quadratic proximal theory (for the case of the Entropic Proximal Decomposition Method).

Our aim is to provide a convergence analysis for an inexact proximal decomposition algorithm using proximal distances, which includes (i) Bregman distances induced by the class of kernels with open domain, (ii) $\phi$ divergence distances, and (iii) log-quadratic distances. Our analysis considers summable errors, which means that infinite sum of all erros is finite.

The rest of the paper is organized as follows. In Section 2 we recall basic notions and properties on setvalued maps, proximal distances, and induced proximal distances. An inexact proximal decomposition method for variational inequalities is presented in Section 3. Then, global convergence of the proposed method is proved in Section 4. Finally, some conclusions are made in Section 5.

\section{BASIC DEFINITIONS}

Given a subset $\mathrm{C} \subset \mathbb{R}^{n}$, we denote by $\operatorname{int}(\mathrm{C})$ its interior and $\overline{\mathrm{C}}$ its closure. A point-to-set mapping (or multifunction) $T: \mathbb{R}^{n} \rightrightarrows \mathbb{R}^{n}$ is an operator which associates with each point $x \in \mathbb{R}^{n}$ a set (possibly empty) $T(x) \subseteq \mathbb{R}^{n}$. The domain and the graph of a point-to-set valued map $T$ are defined as

$$
\begin{aligned}
D(T) & :=\left\{x \in \mathbb{R}^{n}: T(x) \neq \emptyset\right\}, \\
G r(A) & :=\left\{(x, y) \in \mathbb{R}^{n} \times \mathbb{R}^{n}: x \in D(T), y \in T(x)\right\} .
\end{aligned}
$$

A point-to-set operator $T$ is said to be monotone if

$$
\left\langle y^{\prime}-y, x^{\prime}-x\right\rangle \geq 0, \forall y^{\prime} \in T\left(x^{\prime}\right), \forall y \in T(x), \forall x, x^{\prime} \in D(T) .
$$

$T$ is said strictly monotone if the inequality above is strict for all $x, x^{\prime} \in D(T)$ with $x \neq x^{\prime}$. A monotone operator is said to be maximal when its graph is not properly contained in the graph of any other monotone operator.

We shall now present a variant of the definition of the proximal distance and induced proximal distance, introduced by Auslender and Teboulle [3] (see Def. 2.1).

Definition 2.1. A function $d: \mathbb{R}^{n} \times \mathbb{R}^{n} \rightarrow \mathbb{R}_{+} \cup\{+\infty\}$ is called proximal distance with respect to an open nonempty convex set $\mathrm{C} \subset \mathbb{R}^{n}$ if for each $y \in \mathrm{C}$ it satisfies the following properties:

(i) $d(\cdot, y)$ is proper, closed, convex and continuously differentiable on C; 
(ii) $\operatorname{dom} d(\cdot, y) \subset \overline{\mathrm{C}}$ and $\operatorname{dom} \partial_{1} d(\cdot, y)=\mathrm{C}$, where $\partial_{1} d(\cdot, y)$ denotes the classical subgradient map of the function $d(\cdot, y)$ with respect to the first variable;

(iii) $d(\cdot, y)$ is coercive on $\mathbb{R}^{n}$ (i.e., $\left.\lim _{\|u\| \rightarrow \infty} d(u, y)=+\infty\right)$;

(iv) $d(y, y)=0$, wich clearly implies $\nabla_{1} d(y, y)=0$.

We denote by $\mathcal{D}(\mathrm{C})$ the family of functions satisfying the above definition.

Associated to a proximal distance is an induced proximal distance which we define as follows:

Definition 2.2. Given $d \in \mathcal{D}(\mathrm{C})$, a function $H: \mathbb{R}^{n} \times \mathbb{R}^{n} \rightarrow \mathbb{R}_{+} \cup\{+\infty\}$ is called the induced proximal distance to $d$ if there exists $\gamma \in(0,1]$ with $H$ a finite valued on $\mathrm{C} \times \mathrm{C}$ and such that for each $a, b \in \mathrm{C}$, we have

(Ii) $H(a, a)=0$;

(Iii) $\left\langle c-b, \nabla_{1} d(b, a)\right\rangle \leq H(c, a)-H(c, b)-\gamma H(b, a), \quad \forall c \in \mathrm{C}$.

The motivation of the above definition is explained in detail by Auslender and Teboulle (see [2], Def. 2.2). Note that, when $\mathrm{C}=\mathbb{R}^{n}$ and $d(x, y)=2^{-1}\|x-y\|^{2}$, with $d=H$ and $\gamma=1$, then the inequality (Iii) becomes the usual Pythagoras identity.

We write $(d, H) \in \mathcal{F}(\mathrm{C})$ to the proximal and induced proximal distance that satisfies the premises of Definition 2.2 .

We also denote $(d, H) \in \mathcal{F}(\overline{\mathrm{C}})$ if there exists $H$ such that:

(Iiii) $H$ is finite valued on $\overline{\mathrm{C}} \times \mathrm{C}$ satisfying (Ii) and (Iii), for each $c \in \overline{\mathrm{C}}$.

(Iiv) For each $c \in \overline{\mathrm{C}}, H(c, \cdot)$ has level bounded sets on $\mathrm{C}$.

Finally, we write $(d, H) \in \mathcal{F}_{+}(\overline{\mathbf{C}})$ if

(Iv) $(d, H) \in \mathcal{F}(\overline{\mathrm{C}})$.

(Ivi) $\forall y \in \overline{\mathrm{C}}$ and $\forall\left\{y^{k}\right\} \subset \mathrm{C}$ bounded with $\lim _{k \rightarrow+\infty} H\left(y, y^{k}\right)=0$, we have $\lim _{k \rightarrow+\infty} y^{k}=y$.

(Ivii) $\forall y \in \overline{\mathrm{C}}$ and $\forall\left\{y^{k}\right\} \subset \mathrm{C}$ such that $\lim _{k \rightarrow+\infty} y^{k}=y$, we obtain $\lim _{k \rightarrow+\infty} H\left(y, y^{k}\right)=0$.

Several examples of proximal distances which satisfy the above definitions, for example Bregman distances, proximal distances based on $\varphi$-divergences, self-proximal distances, and distances based on second order homogeneous proximal distances, were given by Auslender and Teboulle [3].

The following additional conditions on $H$ will be useful to prove the convergence of the proposed algorithm. Given $(d, H) \in \mathcal{F}_{+}(\overline{\mathrm{C}}), H$ satisfies the following condition:

(Iviii) $\forall c \in \overline{\mathrm{C}}$ and $\forall\left\{y^{k}\right\} \subset \mathrm{C}$ such that $\lim _{k \rightarrow+\infty} y^{k}=y$, we obtain $\lim _{k \rightarrow+\infty} H\left(c, y^{k}\right)=H(c, y)$.

Some examples of proximal distances which satisfy this condition, were shown by Sarmiento et al. [21], Section 7. The main result of the method will be when $(d, H) \in \mathcal{F}_{+}(\overline{\mathrm{C}})$ and the condition (Iviii) is satisfied.

\section{The proximal Decomposition algorithm with proximal distances (PDAPD)}

We are interested in solving the following primal-dual formulation of the $\operatorname{VI}(T, \Omega)$ problem: find $\left(x^{*}, z^{*}, y^{*}\right) \in$ $\overline{\mathrm{C}} \times \overline{\mathrm{K}} \times \mathbb{R}^{m}$ and $g^{*}:=\left(g_{1}^{*}, g_{2}^{*}\right) \in T\left(x^{*}, z^{*}\right)$ such that

$$
\begin{aligned}
\left\langle x-x^{*}, g_{1}^{*}+A^{T} y^{*}\right\rangle+\left\langle z-z^{*}, g_{2}^{*}+B^{T} y^{*}\right\rangle & \geq 0, \forall(x, z) \in \overline{\mathrm{C}} \times \overline{\mathrm{K}}, \\
A x^{*}+B z^{*} & =b .
\end{aligned}
$$

where $\mathrm{C} \subset \mathbb{R}^{n}$ and $\mathrm{K} \subset \mathbb{R}^{p}$ are nonempty open convex sets, $\overline{\mathrm{C}}$ and $\overline{\mathrm{K}}$ denote the closure of $\mathrm{C}$ and $\mathrm{K}$ respectively, $A \in \mathbb{R}^{m \times n}, B \in \mathbb{R}^{m \times p}$ and $b \in \mathbb{R}^{m}$.

Throughout this section we impose the following assumptions for the $\operatorname{VI}(T, \Omega)$ problem. 


\section{Assumptions $\mathcal{A}$.}

$\left(\mathcal{A}_{1}\right)$ The $\operatorname{VI}(T, \Omega)$ problem has a solution.

$\left(\mathcal{A}_{2}\right) D(T) \cap(\mathrm{C} \times \mathrm{K}) \neq \emptyset$.

Remark 3.1. These assumptions were proposed by Auslender and Teboulle (see [1], p. 35), to derive welldefinition of the Entropic Proximal Decomposition Method and also will be used here to ensure that the iterations given by the proposed method are well-defined. The above assumptions are classical conditions given in proximal algorithms for variational inequality problems, see $[1,4,7,8]$.

We will also used the following notations. Let $\mathrm{M}=\overline{\mathrm{C}} \times \overline{\mathrm{K}}$ and set $\bar{T}:=T+\mathcal{N}_{\mathrm{M}}$ where $\mathcal{N}_{\mathrm{M}}$ is the normal cone for the set $\mathrm{M}$, i.e.,

$$
\mathcal{N}_{\mathrm{M}}(w)= \begin{cases}\{v:\langle v, q-w\rangle \leq 0, \forall q \in \mathrm{M}\} & \text { if } w \in \mathrm{M} \\ \emptyset & \text { otherwise, }\end{cases}
$$

where $\langle\cdot, \cdot\rangle$ is the inner product in the product space $\mathbb{R}^{n} \times \mathbb{R}^{p}$. It is well known [19] that the normal cone operator $\mathcal{N}_{\mathrm{M}}: \mathbb{R}^{n} \times \mathbb{R}^{p} \rightrightarrows \mathbb{R}^{n} \times \mathbb{R}^{p}$ is maximal monotone, with dom $\mathcal{N}_{\mathrm{M}}=$ M. Thus, thanks to Assumption $\left(\mathcal{A}_{2}\right)$, one has $\operatorname{dom} T \cap \operatorname{int} \operatorname{dom} \mathcal{N}_{\mathrm{M}}=\operatorname{dom} T \cap(\mathrm{C} \times \mathrm{K}) \neq \emptyset$ and therefore the operator $\bar{T}=T+\mathcal{N}_{\mathrm{M}}$ remains also maximal monotone [20].

Now, we propose an algorithm to solve the problems (3.1) and (3.2). This algorithm is a natural generalization of the Entropic Proximal Decomposition Method (EPDM) proposed by Auslende and Teboulle [1]. Here, we use the generalized proximal distance instead of the logarithmic quadratic proximal distance. It is shown in [3], Section 3, that the logarithmic quadratic proximal distance is a particular case of the generalized proximal distances.

In the proposed algorithm we use the class of proximal distances $\left(d_{0}, H_{0}\right) \in \mathcal{F}_{+}(\overline{\mathrm{C}}),\left(d_{0}^{\prime}, H_{0}^{\prime}\right) \in \mathcal{F}_{+}(\overline{\mathrm{K}})$, satisfying the condition (Iviii) and given $\mu>0, \mu^{\prime}>0$ we define the following functions:

$$
\begin{aligned}
d(x, y) & =d_{0}(x, y)+(\mu / 2)\|x-y\|^{2}, \\
H(x, y) & =H_{0}(x, y)+(\mu / 2)\|x-y\|^{2}, \\
d^{\prime}(x, y) & =d_{0}^{\prime}(x, y)+\left(\mu^{\prime} / 2\right)\|x-y\|^{2}, \\
H^{\prime}(x, y) & =H_{0}^{\prime}(x, y)+\left(\mu^{\prime} / 2\right)\|x-y\|^{2} .
\end{aligned}
$$

It is easy to check that $(d, H) \in \mathcal{F}_{+}(\overline{\mathrm{C}})$ and $\left(d^{\prime}, H^{\prime}\right) \in \mathcal{F}_{+}(\overline{\mathrm{K}})$ (for the same value of $\gamma$ and $\gamma^{\prime}$ respectively) and both satisfy the condition (Iviii).

The algorithm, which will be called Proximal Decomposition Algorithm with Proximal Distances (PDAPD) is as follows:

\section{(PDAPD) Algorithm}

Step 0. Choose two pairs $\left(d_{0}, H_{0}\right) \in \mathcal{F}_{+}(\overline{\mathrm{C}}),\left(d_{0}^{\prime}, H_{0}^{\prime}\right) \in \mathcal{F}_{+}(\overline{\mathrm{K}})$ satisfying the condition (Iviii) and define $(d, H)$, $\left(d^{\prime}, H^{\prime}\right)$ given by (3.3), (3.4) and (3.5), (3.6) respectively.

Take $\left\{\lambda_{k}\right\}$ a sequence of positive scalars to be specified latter. Start with an arbitrary point $\left(x^{0}, z^{0}, y^{0}\right) \in$ $\mathrm{C} \times \mathrm{K} \times \mathbb{R}^{m}$ and generate the sequences $\left\{x^{k}, z^{k}, y^{k}\right\} \subset \mathrm{C} \times \mathrm{K} \times \mathbb{R}^{m}$ and $\left(e_{1}^{k}, e_{2}^{k}\right) \in \mathbb{R}^{n} \times \mathbb{R}^{p}$ as follows:

Step 1. For $k=0,1,2, \ldots$, calculate $p^{k+1} \in \mathbb{R}^{m}$ by

$$
p^{k+1}=y^{k}+\lambda_{k}\left(A x^{k}+B z^{k}-b\right) .
$$

Step 2. Find $\left(x^{k+1}, z^{k+1}\right) \in \mathrm{C} \times \mathrm{K},\left(e_{1}^{k+1}, e_{2}^{k+1}\right) \in \mathbb{R}^{n} \times \mathbb{R}^{p}$ and $g^{k+1}:=\left(g_{1}^{k+1}, g_{2}^{k+1}\right) \in T\left(x^{k+1}, z^{k+1}\right)$ such that

$$
\begin{aligned}
& g_{1}^{k+1}+A^{T} p^{k+1}+\lambda_{k}^{-1} \nabla_{1} d\left(x^{k+1}, x^{k}\right)=e_{1}^{k+1}, \\
& g_{2}^{k+1}+B^{T} p^{k+1}+\lambda_{k}^{-1} \nabla_{1} d^{\prime}\left(z^{k+1}, z^{k}\right)=e_{2}^{k+1},
\end{aligned}
$$

where $\left(e_{1}^{k+1}, e_{2}^{k+1}\right)$ is an approximation error which satisfies some conditions to be specific later. 
Step 3. Compute

$$
y^{k+1}=y^{k}+\lambda_{k}\left(A x^{k+1}+B z^{k+1}-b\right) .
$$

Stopping criterion. If $x^{k+1}=x^{k}, z^{k+1}=z^{k}$ and $y^{k+1}=y^{k}$ then stop. Otherwise do $k:=k+1$, and go to Step 1.

Remark 3.2. As we are interested in the asymptotic convergence of the method, we assume in each iteration that $\left(x^{k+1}, z^{k+1}, y^{k+1}\right) \neq\left(x^{k}, z^{k}, y^{k}\right)$ for each $k=1,2, \ldots$ Indeed, if $\left(x^{k+1}, z^{k+1}, y^{k+1}\right)=\left(x^{k}, z^{k}, y^{k}\right)$ for some $k$, then $\nabla_{1} d\left(x^{k+1}, x^{k}\right)=0$ and $\nabla_{1} d^{\prime}\left(z^{k+1}, z^{k}\right)=0$ and from (3.8) to (3.9) we have that (3.1) and (3.2) hold approximately, that is, $\left(x^{k}, z^{k}, y^{k}\right)$ is an approximate solution of primal-dual formulation of $\operatorname{VI}(T, \Omega)$.

Before we prove the existence of the sequences $\left\{x^{k}, z^{k}, y^{k}\right\}$ and $\left\{e_{1}^{k}, e_{2}^{k}\right\}$ generated by the (PDAPD), we show a more general result of independent interest. For that consider a class of funtions $h: \mathbb{R}^{p} \rightarrow \mathbb{R} \cup\{+\infty\}$ satisfying the following properties:

(i) $h$ is a closed proper convex function with $\operatorname{dom} h$ open,

(ii) $h$ is differentiable on $\operatorname{dom} h$,

(iii) $h_{\infty}(s)=+\infty, \forall s \neq 0$.

Here $h_{\infty}$ denotes the recession function of $h$, see [19] for definition. We denote by $\vartheta$ the class of functions satisfying (i)-(iii).

Remark 3.3. Note that for fixed $\left(x^{k}, z^{k}\right) \in \mathrm{C} \times \mathrm{K}$, the functions $d\left(\cdot, x^{k}\right)$ and $d^{\prime}\left(\cdot, z^{k}\right)$, defined by (3.3) and (3.5) respectively, clearly satisfies properties (i)-(iv).

The existence of the sequences $\left\{x^{k}, z^{k}, y^{k}\right\}$ and $\left\{e_{1}^{k}, e_{2}^{k}\right\}$ will be a consequence of the following general result whose proof is similar to the proof given by Auslender et al. [4], Proposition 2.

Lemma 3.4. Let $h \in \vartheta$. Then,

(1) The gradient mapping $\nabla h$ is onto.

(2) Let $T$ be a maximal monotone map such that $\operatorname{dom} T \cap \operatorname{dom} h \neq \emptyset$ and set

$$
U(x)= \begin{cases}T(x)+\nabla h(x), & \forall x \in \operatorname{dom} T \cap \operatorname{dom} \nabla h \\ \emptyset & \text { otherwise }\end{cases}
$$

Then, there exists at least a solution $x$ of the generalized equation

$$
0 \in U(x)
$$

If in addition, $h$ is supposed to be strictly convex on its domain, then the solution $x$ is unique.

Proof. (1) Let $y \in \mathbb{R}^{p}$ and set $\nu(x)=h(x)-\langle y, x\rangle$. Since $\nu_{\infty}(s)=h_{\infty}(s)-\langle y, s\rangle$ (see [19], Thm. 9.3), we have

$$
\nu_{\infty}(s)=+\infty \quad \forall s \neq 0 .
$$

As a consequence of (3.12), if we minimize $\nu$ on $\mathbb{R}^{p}$, the optimal set is nonempty and since dom $\nu$ is open, each optimal solution $x$ satisfies $\nabla h(x)=y$, so that $\nabla h$ is onto.

(2) Let $\partial g$ be the subdifferential of a closed proper function $g: \mathbb{R}^{p} \rightarrow \mathbb{R} \cup\{+\infty\}$ such that dom Tกint dom $g \neq \emptyset$. It has been proved by [8], Proposition 3, that if $\partial g$ is onto then $T+\partial g$ is onto. From part (1), we have that $\nabla h$ is onto. Then using this result it follows that the generalized equation (3.11) admits at least a solution. If in addition $h$ is strictly convex on its effective domain, then $T+\nabla h$ is strictly monotone which implies uniqueness. 
Due to Remark 3.3 we have to make the following assumption:

$\left(\mathcal{A}_{3}\right)$ The proximal distances $d$ and $d^{\prime}$ have open domains.

Remark 3.5. A large class of proximal distances satisfy the above assumptions. For example

- Separable Bregman distances

$$
d_{h}(x, y):=h(x)-(h(y)+\langle\nabla h(y), x-y\rangle)
$$

induced by $h(x)=\sum_{j=1}^{n} \theta\left(x_{j}\right)$ where $\theta$ can be defined by $\theta(t)=-\log t$ or $\theta(t)=t^{-1}$. It is clear that, in both cases, $d o m d_{h}$ is open.

- Proximal distances based on $\varphi$-divergences defined by

$$
d_{\varphi}(x, y)=\sum_{i=1}^{n} y_{i}^{r} \varphi\left(\frac{x_{i}}{y_{i}}\right) \text { with } r=1,2 .
$$

When $r=1, d_{\varphi}$ is called $\varphi$-divergence proximal distance and $d o m d_{\varphi}$ is open for $\varphi(t)=-\log t+t-1$. When $r=2$ and $\varphi(t)=\mu p(t)+\frac{\nu}{2}(t-1)^{2}$ with $\nu>\mu>0, p(t)=-\log t+t-1, d_{\varphi}$ is called second order homogeneous proximal distance (also known as log-quadratic proximal distance) and clearly dom $d_{\varphi}$ is open.

Proposition 3.6. Assuming the assumptions $\left(\mathcal{A}_{1}\right)-\left(\mathcal{A}_{3}\right)$. For any $\lambda_{k}>0,\left(x^{k}, z^{k}, y^{k}\right) \in C \times K \times \mathbb{R}^{m}, \forall k \geq 0$, there exists a unique point $\left(x^{k+1}, z^{k+1}\right) \in C \times K$ satisfying (3.8) and (3.9) with $g^{k+1} \in T\left(x^{k+1}, z^{k+1}\right)$.

Proof. Let $P_{k}(\cdot):=\lambda_{k}^{-1} \nabla_{1} d\left(\cdot, x^{k}\right)$ and $Q_{k}(\cdot):=\lambda_{k}^{-1} \nabla_{1} d^{\prime}\left(\cdot, z^{k}\right)$. Then $P_{k}$ and $Q_{k}$ are strictly monotone operators because $d$ and $d^{\prime}$ are strictly convex functions (see Defs. 2.1(i) and (3.3)). This implies strict monotonocity of $T_{k}:=T+\left(A^{T} p^{k+1}, B^{T} p^{k+1}\right)+\left(P_{k}, Q_{k}\right)$. Since $T+\left(A^{T} p^{k+1}, B^{T} p^{k+1}\right)$ is maximal monotone and $d, d^{\prime} \in \vartheta$, by Lemma 3.4(2) we have that $T_{k}$ has a zero in $D\left(T_{k}\right)$, which is unique by strict monotonicity. We call this zero $\left(x^{k+1}, z^{k+1}\right)$. Thus, it is clear that (3.8) and (3.9) hold. Now, we have to show that $\left(x^{k+1}, z^{k+1}\right)$ belongs to $\mathrm{C} \times \mathrm{K}$. By Definition 2.1(ii), $D\left(T_{k}\right)=D(T) \cap(\mathrm{C} \times \mathrm{K})$, and since $\left(x^{k+1}, z^{k+1}\right) \in D\left(T_{k}\right)$ we obtain that $\left(x^{k+1}, z^{k+1}\right) \in$ $\mathrm{C} \times \mathrm{K}$.

\section{Global Convergence}

In this section, under appropriate assumptions, we establish the global convergence of the PDAPD.

\section{Assumptions $\mathcal{B}$.}

$\left(\mathcal{B}_{1}\right)$ Given the parameters $\mu>0, \mu^{\prime}>0$, defined in (3.3) and (3.5) respectively, the sequence $\left\{\lambda_{k}\right\}$ satisfies

$$
\eta<\lambda_{k}<\bar{c}-\eta
$$

where $\eta \in(0, \bar{c} / 2)$ with $\bar{c}:=\min \left\{\frac{\sqrt{\gamma \mu}}{2\|A\|}, \frac{\sqrt{\gamma^{\prime} \mu^{\prime}}}{2\|B\|}\right\}$ and $\gamma, \gamma^{\prime}$ are positive constants related to $d$ and $d^{\prime}$, respectively, in Definition 2.2(Iii).

$\left(\mathcal{B}_{2}\right)$ Given the sequence $\left\{\left(x^{k}, z^{k}\right)\right\}$ generated by (PDAPD) algorithm, we assume the following additional conditions on the sequences of errors $\left\{e_{1}^{k}\right\},\left\{e_{2}^{k}\right\}$ :

$$
\begin{array}{cc}
\sum_{k=0}^{\infty}\left\langle x^{k}, e_{1}^{k}\right\rangle<+\infty, & \sum_{k=0}^{\infty}\left\langle z^{k}, e_{2}^{k}\right\rangle<+\infty \\
\sum_{k=0}^{\infty}\left\|e_{1}^{k}\right\|<+\infty, & \sum_{k=0}^{\infty}\left\|e_{2}^{k}\right\|<+\infty
\end{array}
$$


Remark 4.1. Assumption $\left(\mathcal{B}_{1}\right)$ will be used to ensure the convergence of the method. Observe that the interval $(\eta, \bar{c}-\eta)$ depends of $\mu$ and $\mu^{\prime}$ which are arbitrary.

The condition $\left(\mathcal{B}_{2}\right)$ is a kind of condition for the error sequences that were given in $[10,14,22]$ and similar forms can be found for example in [16]. Condition (4.2) might appear somewhat unnatural since it involves the iterates $x^{k}$ and $z^{k}$ which are a priori unknown. However, it was noticed in [1], p. 10, that (4.2) is easily enforcable in practice, and also implied by the more easily verified condition

$$
\sum_{k=0}^{\infty}\left\|e_{1}^{k}\right\|\left\|x^{k}\right\|<+\infty, \quad \sum_{k=0}^{\infty}\left\|e_{2}^{k}\right\|\left\|z^{k}\right\|<+\infty
$$

which is satisfied in particular when $e_{1}^{k}=0, e_{2}^{k}=0$ for each $k$ or when $\mathrm{C}$ and $\mathrm{K}$ or $\operatorname{dom} T$ is bounded (in addition, with (4.3)).

The subsequent convergence analysis follows a line of argument similar to that given in [1]. The first result is a classical property in proximal point algorithms.

Lemma 4.2 ([9], Lem. 3.1). Let $F: \mathbb{R}^{m} \rightarrow(-\infty,+\infty]$ be a closed proper convex function, $\tau>0$ and define:

$$
u^{k+1}=\underset{u \in \mathbb{R}^{m}}{\arg \min }\left\{F(u)+(1 /(2 \tau))\left\|u-u^{k}\right\|^{2}\right\} .
$$

Then for any integer $k \geq 0$,

$$
2 \tau\left[F\left(u^{k+1}\right)-F(u)\right] \leq\left\|u^{k}-u\right\|^{2}-\left\|u^{k+1}-u\right\|^{2}-\left\|u^{k+1}-u^{k}\right\|^{2}, \forall u \in \mathbb{R}^{m} .
$$

The next Proposition will play an important role in the analysis of the proposed algorithm. We will use the following useful notation

$$
\Delta_{k}(x, z)=H\left(x, x^{k}\right)-H\left(x, x^{k+1}\right)-\gamma H\left(x^{k+1}, x^{k}\right)+H^{\prime}\left(z, z^{k}\right)-H^{\prime}\left(z, z^{k+1}\right)-\gamma^{\prime} H^{\prime}\left(z^{k+1}, z^{k}\right) .
$$

Proposition 4.3. Let $\left(d_{0}, H_{0}\right) \in \mathcal{F}(\bar{C}),\left(d_{0}^{\prime}, H_{0}^{\prime}\right) \in \mathcal{F}(\bar{K})$ be proximal and induced proximal distances. Suppose that the assumptions $\left(\mathcal{A}_{1}\right)-\left(\mathcal{A}_{3}\right)$ are satisfied. Then, for any $(x, z, y) \in \bar{C} \times \bar{K} \times \mathbb{R}^{m}$ and $g=\left(g_{1}, g_{2}\right) \in T(x, z)$ the following inequalities hold:

$$
\begin{aligned}
& \lambda_{k}\left(\left\langle g_{1}+A^{T} p^{k+1}, x^{k+1}-x\right\rangle\right.\left.+\left\langle g_{2}+B^{T} p^{k+1}, z^{k+1}-z\right\rangle\right) \\
& \leq \Delta_{k}(x, z)+\lambda_{k}\left(\left\langle x^{k+1}-x, e_{1}^{k+1}\right\rangle+\left\langle z^{k+1}-z, e_{2}^{k+1}\right\rangle\right) \\
& 2 \lambda_{k}\left\langle y^{k+1}-p^{k+1}, A x^{k}+B z^{k}-b\right\rangle \leq\left\|y^{k}-y^{k+1}\right\|^{2}-\left\|p^{k+1}-y^{k+1}\right\|^{2}-\left\|p^{k+1}-y^{k}\right\|^{2} \\
& 2 \lambda_{k}\left\langle y-y^{k+1}, A x^{k}+B z^{k}-b\right\rangle \leq\left\|y^{k}-y\right\|^{2}-\left\|y^{k+1}-y\right\|^{2}-\left\|y^{k+1}-y^{k}\right\|^{2} .
\end{aligned}
$$

Proof. By Proposition 3.6, one has $\left(x^{k+1}, z^{k+1}\right) \in \mathrm{C} \times \mathrm{K}$, and $\left(g_{1}^{k+1}, g_{2}^{k+2}\right) \in T\left(x^{k+1}, z^{k+1}\right)$. Then, using the monotonicity of $T$, we obtain that for any $(x, z) \in \overline{\mathrm{C}} \times \overline{\mathrm{K}}$ and $\left(g_{1}, g_{2}\right) \in T(x, z)$ :

$$
0 \leq\left\langle x-x^{k+1}, g_{1}-g_{1}^{k+1}\right\rangle+\left\langle z-z^{k+1}, g_{2}-g_{2}^{k+1}\right\rangle,
$$

then using (3.8) and (3.9), we obtain

$$
\begin{aligned}
\lambda_{k}\left(\left\langle g_{1}+A^{T} p^{k+1}, x^{k+1}-x\right\rangle+\right. & \left.\left\langle g_{2}+B^{T} p^{k+1}, z^{k+1}-z\right\rangle\right) \\
\leq & \left\langle x-x^{k+1}, \nabla_{1} d\left(x^{k+1}, x^{k}\right)\right\rangle-\lambda_{k}\left\langle x-x^{k+1}, e_{1}^{k+1}\right\rangle \\
& +\left\langle z-z^{k+1}, \nabla_{1} d^{\prime}\left(z^{k+1}, z^{k}\right)\right\rangle-\lambda_{k}\left\langle z-z^{k+1}, e_{2}^{k+1}\right\rangle .
\end{aligned}
$$

Using Defintion 2.2(Iii), with $c=x, b=x^{k+1}, a=x^{k}$ and $c^{\prime}=z, b^{\prime}=z^{k+1}, a^{\prime}=z^{k}$, after adding the inequalities and considering the notation (4.5), we obtain (i). 
To prove (ii) and (iii) note that Steps 1 and 3 can be written equivalently as:

$$
\begin{aligned}
& p^{k+1}=\underset{v \in \mathbb{R}^{m}}{\arg \min }\left\{-\left\langle v, A x^{k}+B z^{k}-b\right\rangle+\left(1 / 2 \lambda_{k}\right)\left\|v-y^{k}\right\|^{2}\right\}, \\
& y^{k+1}=\underset{v \in \mathbb{R}^{m}}{\arg \min ^{m}}\left\{-\left\langle v, A x^{k+1}+B z^{k+1}-b\right\rangle+\left(1 / 2 \lambda_{k}\right)\left\|y-y^{k}\right\|^{2}\right\} .
\end{aligned}
$$

Then, using Lemma 4.2 twice with $\tau=\lambda_{k}, F(u)=-\left\langle u, A x^{k}+B z^{k}-b\right\rangle$ and $F(u)=-\left\langle u, A x^{k+1}+B z^{k+1}-b\right\rangle$ respectively, the first and second equations above respectively yield the desired inequalities (ii) and (iii).

Before proving our main convergence result, we will also need the following technical result.

Lemma 4.4 ([18], Lem. 2). Let $\left\{\alpha_{k}\right\}$ and $\left\{\beta_{k}\right\}$ be nonnegative sequences of real numbers such that $\alpha_{k+1} \leq$ $\alpha_{k}+\beta_{k}$, for all $k$ and $\sum_{k=0}^{\infty} \beta_{k}<+\infty$. Then, the sequence $\left\{\alpha_{k}\right\}$ converges.

Remark 4.5. Throughout the rest of this paper, we denote $w^{k}=\left(x^{k}, z^{k}, y^{k}\right), w^{*}=\left(x^{*}, z^{*}, y^{*}\right), w=(x, z, y)$, $s=(l, q, r) \in \mathbb{R}^{n} \times \mathbb{R}^{p} \times \mathbb{R}^{m}$ and we define the function $\hat{H}: X \times X \rightarrow \mathbb{R}_{+} \cup\{+\infty\}$ by

$$
\hat{H}(w, s)=\hat{H}((x, z, y),(l, q, r))=H(x, l)+H^{\prime}(z, q)+(1 / 2)\|y-r\|^{2},
$$

where $X=\mathbb{R}^{n} \times \mathbb{R}^{p} \times \mathbb{R}^{m}, H$ and $H^{\prime}$ are defined in (3.4) and (3.6) respectively.

We can now state and prove our main convergence result for PDAPD.

Theorem 4.6. Consider the variational inequality problem $V I(T, \Omega)$ and suppose that assumptions $\left(\mathcal{A}_{1}\right)$ to $\left(\mathcal{A}_{3}\right)$ and $\left(\mathcal{B}_{1}\right),\left(\mathcal{B}_{2}\right)$ hold. Let $\left(d_{0}, H_{0}\right) \in \mathcal{F}_{+}(\bar{C}),\left(d_{0}^{\prime}, H_{0}^{\prime}\right) \in \mathcal{F}_{+}(\bar{K})$ be proximal and induced proximal distances satisfying the condition (Iviii), and let $\left\{\left(x^{k}, z^{k}, y^{k}\right)\right\}$ be the sequence generated by PDAPD, then the sequence $\left\{\left(x^{k}, z^{k}, y^{k}\right)\right\}$ globally converges to $\left(x^{*}, z^{*}, y^{*}\right)$, with $\left(x^{*}, z^{*}\right)$ solution of $V I(T, \Omega)$.

Proof. Let $w^{*}:=\left(x^{*}, z^{*}, y^{*}\right) \in \mathrm{C} \times \mathrm{K} \times \mathbb{R}^{m}$ with $g^{*}=\left(g_{1}^{*}, g_{2}^{*}\right) \in T\left(x^{*}, z^{*}\right)$ be a solution of (3.1) and (3.2) and let $\left\{\left(x^{k}, z^{k}, y^{k}\right)\right\}$ be the sequence produced by PDAPD. Then from (3.1) it follows that

$$
\left\langle x^{k+1}-x^{*}, g_{1}^{*}+A^{T} y^{*}\right\rangle+\left\langle z^{k+1}-z^{*}, g_{2}^{*}+B^{T} y^{*}\right\rangle \geq 0 .
$$

Using Proposition 4.3(i) at $(x, z, y):=\left(x^{*}, z^{*}, y^{*}\right)$ and (4.5) we have:

$$
\begin{aligned}
\lambda_{k}\left(\left\langle g_{1}^{*}+A^{T} p^{k+1}, x^{k+1}-x^{*}\right\rangle\right. & \left.+\left\langle g_{2}^{*}+B^{T} p^{k+1}, z^{k+1}-z^{*}\right\rangle\right) \\
& \leq \Delta_{k}\left(x^{*}, z^{*}\right)+\lambda_{k}\left(\left\langle x^{k+1}-x^{*}, e_{1}^{k+1}\right\rangle+\left\langle z^{k+1}-z^{*}, e_{2}^{k+1}\right\rangle\right) .
\end{aligned}
$$

Adding (4.7) and (4.8), and using $A x^{*}+B z^{*}=b$ we obtain

$$
\lambda_{k}\left\langle p^{k+1}-y^{*}, A x^{k+1}+B z^{k+1}-b\right\rangle \leq \Delta_{k}\left(x^{*}, z^{*}\right)+\lambda_{k}\left(\left\langle x^{k+1}-x^{*}, e_{1}^{k+1}\right\rangle+\left\langle z^{k+1}-z^{*}, e_{2}^{k+1}\right\rangle\right),
$$

replacing the notation of $\Delta_{k}\left(x^{*}, z^{*}\right)$ (see (4.5)) and after rearranging, we obtain

$$
\begin{aligned}
H\left(x^{*}, x^{k+1}\right)+H^{\prime}\left(z^{*}, z^{k+1}\right) \leq & H\left(x^{*}, x^{k}\right)+H^{\prime}\left(z^{*}, z^{k}\right)-\gamma H\left(x^{k+1}, x^{k}\right)-\gamma^{\prime} H^{\prime}\left(z^{k+1}, z^{k}\right) \\
& +\lambda_{k}\left\langle y^{*}-p^{k+1}, A x^{k+1}+B z^{k+1}-b\right\rangle \\
& +\lambda_{k}\left(\left\langle x^{k+1}-x^{*}, e_{1}^{k+1}\right\rangle+\left\langle z^{k+1}-z^{*}, e_{2}^{k+1}\right\rangle\right) .
\end{aligned}
$$

Now, adding the inequalities (ii) and (iii) of Proposition 4.3 for the point $y=y^{*}$ we get

$$
\begin{aligned}
(1 / 2)\left\|y^{*}-y^{k+1}\right\|^{2} \leq & (1 / 2)\left\|y^{*}-y^{k}\right\|^{2}-(1 / 2)\left\|p^{k+1}-y^{k+1}\right\|^{2}-(1 / 2)\left\|p^{k+1}-y^{k}\right\|^{2} \\
& +\lambda_{k}\left\langle p^{k+1}-y^{k+1}, A x^{k}+B z^{k}-b\right\rangle \\
& +\lambda_{k}\left\langle y^{k+1}-y^{*}, A x^{k+1}+B z^{k+1}-b\right\rangle .
\end{aligned}
$$


For a vector $w=(x, z, y)$, adding (4.9), (4.10) and considering (4.6), (see Remark 4.5), we obtain

$$
\begin{aligned}
\hat{H}\left(w^{*}, w^{k+1}\right) \leq & \hat{H}\left(w^{*}, w^{k}\right)-\left(\gamma H\left(x^{k+1}, x^{k}\right)+\gamma^{\prime} H^{\prime}\left(z^{k+1}, z^{k}\right)\right) \\
& -(1 / 2)\left(\left\|p^{k+1}-y^{k+1}\right\|^{2}+\left\|p^{k+1}-y^{k}\right\|^{2}\right)+\rho_{k} \\
& +\lambda_{k}\left(\left\langle x^{k+1}-x^{*}, e_{1}^{k+1}\right\rangle+\left\langle z^{k+1}-z^{*}, e_{2}^{k+1}\right\rangle\right)
\end{aligned}
$$

where,

$$
\rho_{k}=\lambda_{k}\left\langle y^{k+1}-p^{k+1}, A\left(x^{k+1}-x^{k}\right)+B\left(z^{k+1}-z^{k}\right)\right\rangle .
$$

Now, using (3.7) and (3.10) as given in Step 1 and Step 3 of (PDAPD), it follows that

$$
\begin{aligned}
\rho_{k} & =\lambda_{k}^{2}\left\langle A\left(x^{k+1}-x^{k}\right)+B\left(z^{k+1}-z^{k}\right), A\left(x^{k+1}-x^{k}\right)+B\left(z^{k+1}-z^{k}\right)\right\rangle . \\
& =\lambda_{k}^{2}\left\|A\left(x^{k+1}-x^{k}\right)+B\left(z^{k+1}-z^{k}\right)\right\|^{2} \\
& \leq 2 \lambda_{k}^{2}\left(\|A\|^{2}\left\|x^{k+1}-x^{k}\right\|^{2}+\|B\|^{2}\left\|z^{k+1}-z^{k}\right\|^{2}\right) .
\end{aligned}
$$

Using this estimation for $\rho_{k}$ in (4.11), and from definitions of $H(\cdot, \cdot)$ and $H^{\prime}(\cdot, \cdot)$ (see 3.4 and 3.6 respectively), we obtain

$$
\begin{aligned}
\hat{H}\left(w^{*}, w^{k+1}\right) \leq & \hat{H}\left(w^{*}, w^{k}\right)-\left[\gamma H_{0}\left(x^{k+1}, x^{k}\right)+(1 / 2)\left(\gamma \mu-4 \lambda_{k}^{2}\|A\|^{2}\right)\left\|x^{k+1}-x^{k}\right\|^{2}\right] \\
& -\left[\gamma^{\prime} H_{0}^{\prime}\left(z^{k+1}, z^{k}\right)+(1 / 2)\left(\gamma^{\prime} \mu^{\prime}-4 \lambda_{k}^{2}\|B\|^{2}\right)\left\|z^{k+1}-z^{k}\right\|^{2}\right] \\
& -(1 / 2)\left(\left\|p^{k+1}-y^{k+1}\right\|^{2}+\left\|p^{k+1}-y^{k}\right\|^{2}\right) \\
& +\lambda_{k}\left(\left\langle x^{k+1}-x^{*}, e_{1}^{k+1}\right\rangle+\left\langle z^{k+1}-z^{*}, e_{2}^{k+1}\right\rangle\right) .
\end{aligned}
$$

By assumption $\left(\mathcal{B}_{1}\right)$, we obtain

$$
\hat{H}\left(w^{*}, w^{k+1}\right) \leq \hat{H}\left(w^{*}, w^{k}\right)+(\bar{c}-\eta)\left(\left\langle x^{k+1}-x^{*}, e_{1}^{k+1}\right\rangle+\left\langle z^{k+1}-z^{*}, e_{2}^{k+1}\right\rangle\right),
$$

then using (4.13) and assumption $\left(\mathcal{B}_{2}\right)$, we get

$$
w^{k} \in L_{\hat{H}}\left(w^{*}, \bar{\alpha}\right):=\left\{w: \hat{H}\left(w^{*}, w\right) \leq \bar{\alpha}\right\}, \forall k,
$$

where

$$
\bar{\alpha}=\hat{H}\left(w^{*}, w^{0}\right)+\sum_{k=0}^{\infty}(\bar{c}-\eta)\left(\left\langle x^{k+1}-x^{*}, e_{1}^{k+1}\right\rangle+\left\langle z^{k+1}-z^{*}, e_{2}^{k+1}\right\rangle\right)
$$

since $H\left(x^{*}, \cdot\right)$ and $H^{\prime}\left(z^{*}, \cdot\right)$ satisfy Definition 2.2(Iiv), we have that $\left\{w^{k}\right\}$ is bounded.

Moreover from (4.13), assumption $\left(\mathcal{B}_{2}\right)$ and Lemma 4.4 , we obtain that $\left\{\hat{H}\left(w^{*}, w^{k}\right)\right\}$ converges, i.e., there exists $\beta \geq 0$ such that

$$
\lim _{k \rightarrow+\infty} \hat{H}\left(w^{*}, w^{k}\right)=\beta .
$$

Therefore, taking limit in (4.9) when $k \rightarrow+\infty$, we obtain (since $\lambda_{k}$ satisfies Assumption $\left(\mathcal{B}_{1}\right)$ )

$$
\begin{aligned}
H_{0}\left(x^{k+1}, x^{k}\right) & \rightarrow 0,\left\|x^{k+1}-x^{k}\right\| \rightarrow 0, \\
H^{\prime}\left(z^{k+1}, z^{k}\right) & \rightarrow 0,\left\|z^{k+1}-z^{k}\right\| \rightarrow 0, \\
\left\|p^{k+1}-y^{k+1}\right\| & \rightarrow 0,\left\|p^{k+1}-y^{k}\right\| \rightarrow 0 .
\end{aligned}
$$

Let $\bar{w}=(\bar{x}, \bar{z}, \bar{y})$ be an arbitrary cluster point of the bounded sequence $\left\{w^{k}\right\}$ and let $\left\{w^{k}\right\}_{k \in K}$ be a subsequence convergent to $\bar{w}$. We now show that $\bar{w}$ solves (3.1) and (3.2). First, note that from (4.15) it follows that

$$
\begin{aligned}
& \lim _{k \rightarrow \infty, k \in K} x^{k+1}=\bar{x}, \quad \lim _{k \rightarrow \infty, k \in K} z^{k+1}=\bar{z}, \\
& \lim _{k \rightarrow \infty, k \in K} p^{k+1}=\bar{y}, \quad \lim _{k \rightarrow \infty, k \in K} y^{k+1}=\bar{y} .
\end{aligned}
$$


Then, since $\lambda_{k}>\eta>0$, passing limit on the subsequences in (i) of Proposition 4.3 it follows that

$$
\left\langle g_{1}+A^{T} \bar{y}, \bar{x}-x\right\rangle+\left\langle g_{2}+B^{T} \bar{y}, \bar{z}-z\right\rangle \leq 0, \quad \forall x \in \overline{\mathrm{C}}, z \in \overline{\mathrm{K}},\left(g_{1}, g_{2}\right) \in T(x, z) .
$$

Likewise, passing limit on the subsequence in inequality (iii) of Proposition 4.3 we get

$$
\langle y-\bar{y}, A \bar{x}+B \bar{z}-b\rangle \leq 0, \quad \forall y \in \mathbb{R}^{m},
$$

and thus it also follows from (4.18) that $A \bar{x}+B \bar{z}=b$. Furthermore, since the sequence produced by PDAPD satisfies $\left(x^{k}, z^{k}\right) \in \mathrm{C} \times \mathrm{K}, \forall k$, then due to convexity of $\mathrm{C}$ and $\mathrm{K}$ passing to the limit one has $(\bar{x}, \bar{z}) \in \overline{\mathrm{C}} \times \overline{\mathrm{K}}$ and so $\bar{w} \in \overline{\mathrm{C}} \times \overline{\mathrm{K}} \times \mathbb{R}^{m}$.

Let $P$ be the multivalued map defined by $P(x, z):=\bar{T}(x, z)+\left(A^{T} \bar{y}, B^{T} \bar{y}\right)$. Then, since $\bar{T}$ is maximal monotone, $P$ is also maximal monotone. Due that for all $(u, v) \in \mathcal{N}_{\mathbb{M}}(x, z)$ one has

$$
\langle u, \bar{x}-x\rangle+\langle v, \bar{z}-z\rangle \leq 0
$$

then from (4.17) we obtain

$$
\left\langle\left(g_{1}+A^{T} \bar{y}+u\right)-0, x-\bar{x}\right\rangle+\left\langle\left(g_{2}+B^{T} \bar{y}+v\right)-0, z-\bar{z}\right\rangle \geq 0,
$$

which shows, by the maximal monotonicity of $P$, that $(0,0) \in P(\bar{x}, \bar{z})$. As a consequence, there exists $\left(\bar{g}_{1}, \bar{g}_{2}\right) \in$ $T(\bar{x}, \bar{z})$ and $(\bar{u}, \bar{v}) \in \mathcal{N}_{\mathrm{M}}(\bar{x}, \bar{z})$ such that

$$
\bar{g}_{1}+\bar{u}+A^{T} \bar{y}=0, \quad \bar{g}_{2}+\bar{v}+B^{T} \bar{z}=0,
$$

and therefore we have $\forall(x, z) \in \mathrm{M}$

$$
\left\langle\bar{g}_{1}+A^{T} \bar{y}, x-\bar{x}\right\rangle+\left\langle\bar{g}_{2}+B^{T} \bar{z}, z-\bar{z}\right\rangle=-\langle\bar{u}, x-\bar{x}\rangle-\langle\bar{v}, z-\bar{z}\rangle \geq 0,
$$

and due that $A \bar{x}+B \bar{z}=b,(\bar{x}, \bar{z}) \in \overline{\mathrm{C}} \times \overline{\mathrm{K}}$, we can thus conclude that $\bar{w}=(\bar{x}, \bar{z}, \bar{y})$ is a solution of (3.1) and $(3.2)$.

Finally, we will prove that $\left\{w^{k}\right\}$ converges to $\bar{w}$. Indeed, due to (4.16) holds, from Definition 2.2(Ivii), we obtain

$$
\lim _{k \rightarrow \infty, k \in K} H\left(\bar{x}, x^{k}\right)=0, \quad \lim _{k \rightarrow \infty, k \in K} H^{\prime}\left(\bar{z}, z^{k}\right)=0, \quad \lim _{k \rightarrow \infty, k \in K}(1 / 2)\left\|y^{k}-\bar{y}\right\|^{2}=0,
$$

thus, from (4.6) (see Rem. 4.5)

$$
\lim _{k \rightarrow \infty, k \in K} \hat{H}\left(\bar{w}, w^{k}\right)=0,
$$

by (4.14), substituting $w^{*}$ by $\bar{w}$, we obtain that $\left\{\hat{H}\left(\bar{w}, w^{k}\right)\right\}$ converges and as there exists a subsequence which converges to zero, it follows that the whole sequence converges, that is,

$$
\lim _{k \rightarrow+\infty} \hat{H}\left(\bar{w}, w^{k}\right)=0 .
$$

Finally, from (4.6) and using Definition 2.2(Ivi), for $H(\bar{x}, \cdot)$ and $H^{\prime}(\bar{z}, \cdot)$, we obtain

$$
\lim _{k \rightarrow+\infty} w^{k}=\bar{w} .
$$


Thus, we obtain the result.

\section{Concluding Remarks}

- Using the concept of proximal distances introduced by Auslender and Teboulle [3], we introduce an inexact proximal decomposition algorithm to solve monotone variational inequality problems with a given separable structure. The use of proximal distances in the (PDAPD) generalize the works of Chen and Teboulle and the Entropic Proximal Decomposition Method proposed in [1].

- Assuming assumptions $\left(\mathcal{A}_{1}\right)-\left(\mathcal{A}_{3}\right)$ and $\left(\mathcal{B}_{1}\right),\left(\mathcal{B}_{2}\right)$ we prove that the sequence generated by the (PDAPD) globally converges to the unique solution of the problem $\operatorname{VI}(T, \Omega)$.

- The (PDAPD) was motivated from the previous work [21] where we introduced a Proximal Multiplier Algorithm with Proximal Distances (PMAPD) to solve convex optimization problems with a given separable structure. However, these algorithms are different in their inexact versions, so the (PDAPD), introduced in this paper, may be applied to solve convex optimization problems with a given separable structure.

- It would be interesting for a future research prove the convergence and the rate of convergence of the proposed method under weaker assumptions, specially the ones associated to other errors.

- Another future research may be investigate a similar algorithm in the spirit of the ADMM algorithm.

Acknowledgements. The first and third authors wish to thank at Callao National University and INNOVATE-PERÚ by supporting research through the CONVENIO 460-INNOVATEPERU-BRI-2015-Perú. The work of the second author was supported by the National Council for Scientific and Technological Development (CNPq) and by grant E26/200.209/2017, Rio de Janeiro Research Foundation (FAPERJ).

\section{REFERENCES}

[1] A. Auslender and M. Teboulle, Entropic proximal decomposition methods for convex programs and variational inequalities. Math. Program. Ser. A 91 (2001) 33-47.

[2] A. Auslender and M. Teboulle, Interior projection-like methods for monotone variational inequalities. Math. Program. Ser. A 104 (2005) 39-68.

[3] A. Auslender and M. Teboulle, Interior gradient and proximal methods for convex and conic optimization. SIAM J. Optim. 16 (2006) 697-725.

[4] A. Auslender, M. Teboulle and S. Ben-Tiba, A logarithmic-quadratic proximal method for variational inequalities. Comput. Optim. Appl. 12 (1999) 31-40.

[5] A. Auslender, M. Teboulle and S. Ben-Tiba, Interior proximal and multiplier methods based on second order homogeneous functionals. Math. Oper. Res. 24 (1999) 645-668.

[6] D.P. Bertsekas and E.M. Gafni, Projection method for variational inequalities with applications to the traffic assignment problem. Math. Program. Study 17 (1982) 139-159.

[7] R.S. Burachik and J. Dutta, Inexact proximal point methods for variational inequality problems. SIAM J. Optim. 20 (1998) 2653-2678.

[8] R.S. Burachik and A.N. Iusem, A generalized proximal point algorithm for the variational inequality problem in a Hilbert space. SIAM J. Optim. 8 (1998) 197-216.

[9] G. Chen and M. Teboulle, A proximal-based decomposition method for convex minimization problems. Math. Program. Ser. A 64 (1994) 81-101.

[10] J. Eckstein, Approximate iterations in Bregman-function-based proximal algorithms. Math. Program. 83 (1998) $113-123$.

[11] M. Fortin and R. Glowinski, Augmented Lagrangian Methods: Applications to the Solution of Boundary-Valued Problems. North-Holland, Amsterdam (1983).

[12] D. Gabay, Chapter IX applications of the method of multipliers to variational inequalities. Stud. Math. Appl. 15 (1983) 299-331.

[13] R. Glowinski and P. Le Tallec, Augmented Lagrangian and Operator-Splitting Methods in Nonlinear Mechanics. SIAM Studies in Applied Mathematics, Philadelphia, PA (1989).

[14] A. Kaplan and R. Tichatschke, On inexact generalized proximal methods with a weakened error tolerance criterion. Optimization 53 (2004) 3-17.

[15] M. Li and X.M. Yuan, An improved proximal-based decomposition method for structured monotone variational inequalities. Appl. Math. Mech. 28 (2007) 1659-1668.

[16] Z.Q. Luo and P. Tseng, Error bounds and convergence analysis of feasible descent methods: a general approach. Ann. Oper. Res. 46 (1993) 157-178. 
[17] A. Nagurney, Network Economics: A Variational Inequality Approach. Springer Science \& Business Media 10 (2013).

[18] B.T. Polyak, Introduction to Optimization. Optimization Software, Publications Division, New York, NY (1987).

[19] R.T. Rockafellar, Convex Analysis. Princeton University Press, Princeton, NJ (1970).

[20] R.T. Rockafellar, On the maximality of sums of nonlinear monotone operators. Trans. Am. Math. Soc. 149 (1970) $75-88$.

[21] O. Sarmiento, E.A. Papa Quiroz and P.R. Oliveira, A proximal multiplier method for separable convex minimization. Optimization 65 (2016) 501-537.

[22] M.V. Solodov and B.F. Svaiter, An inexact hybrid generalized proximal point algorithm and some new results on the theory of Bregman functions. Math. Oper. Res. 25 (2000) 214-230.

[23] M. Tao and X.M. Yuan, On the $O(1 / t)$ convergence rate of alternating direction method with logarithmic-quadratic proximal regularization. SIAM J. Optim. 22 (2012) 1431-1448. 